\title{
Anxiety symptoms as precursors of major depression and suicidal ideation
}

Philip J. Batterham, Ph.D. ${ }^{1}$, Helen Christensen, Ph.D. ${ }^{2}$ and Alison L. Calear, Ph.D. ${ }^{1}$

${ }^{1}$ Centre for Mental Health Research, The Australian National University, Canberra, Australia

${ }^{2}$ Black Dog Institute, The University of New South Wales, Sydney, Australia

Corresponding author:

Philip Batterham

Centre for Mental Health Research

Building 63, Eggleston Road

The Australian National University

Canberra ACT 0200 AUSTRALIA

Tel.: +61261251031

Fax: +61261250733

Email: philip.batterham@anu.edu.au

Word count: 3,984

Running head: Anxiety preceding depression and suicidal ideation

Key words: anxiety, depression, suicidal ideation, population attributable risk, prevention

programs

Acknowledgements: Funding for Waves 1 and 2 of the PATH study was provided by National Health and Medical Research Council (NHMRC) Program Grant 179805 (CIs Jorm, Christensen, Rodgers, Easteal and Anstey) and Unit Grant No. 973302. Wave 3 funding (CIs Anstey, Christensen, Mackinnon, Easteal, Butterworth ) was provided by NHMRC Project grant No. 157125. PB is funded by NHMRC Early Career Fellowship 1035262. HC is funded by NHMRC Fellowship 525411. AC is funded by NHMRC Early Career Fellowship 1013199. We gratefully acknowledge the participants in this study, Patricia Jacomb, Karen Maxwell and PATH interviewers for their assistance. The authors declare no conflict of interest. 


\section{Abstract}

Background: Relative to depression symptoms, the role of anxiety symptoms in the development of depression and suicidal ideation has not been well established. This study aimed to identify the anxiety and depression symptoms that confer the greatest amount of risk for depression and suicidal ideation at the population level.

Method: The PATH through Life study is an Australian community-based longitudinal cohort study of 7,485 younger, middle-aged and older adults. Adjusted population attributable risk (PAR) for incident depression and suicidal ideation after four years was assessed for 18 symptoms of anxiety and depression.

Results: Anxiety symptoms contributed greater risk overall to both depression (45\%) and suicidal ideation (23\%) incidence than depression symptoms (35\% and $16 \%$ respectively). Anxiety symptoms had largest PARs among younger age groups.

Conclusions: Prevention programs for depression and suicide should aim to reduce anxiety symptoms in addition to depression symptoms, and target individuals reporting symptoms such as worrying or irritability. 
Subclinical depression is known to be a strong risk factor for subsequent major depression [1;2] and thoughts of suicide [3]. However, the role of anxiety symptoms in the development of depression [4] and suicidal ideation [3] is less clear. Although there may be complex etiological interactions between anxiety and depression [5], it is known that anxiety disorders have a high comorbidity with depression [6] and that anxiety disorders occur prior to the onset of depressive disorders in many individuals [7]. Of particular concern is the finding that the course of depression that is comorbid with anxiety or other disorders tends to be more severe than that for depression alone [8]. There is also considerable evidence that individuals with anxiety disorders are at increased risk of suicidal ideation $[9 ; 10]$ and suicide attempt [9; 11], particularly among those with comorbid anxiety and depression [12-14]. However, the comparative predictive value of anxiety symptoms relative to depression symptoms for subsequent development of caseness for depression and suicidal ideation has not been well established.

A standard strategy to maximise the effectiveness of prevention efforts for any disorder is to target programs to those individuals who are at higher risk for disorder and to modify the specific risk factors that confer the most risk of developing disorders across the population. It is therefore important to identify the factors that are likely to make the most impact on prevalence rates in the population. Interventions that target the symptoms that confer the greatest vulnerability may be more successful than those that aim to reduce overall symptom levels more generally [see 4]. Using measures of population attributable risk, it is possible to quantify the risk of depression and suicidal ideation that are attributable to single or multiple factors $[15 ; 16]$. Such measures take into account both the prevalence of a risk factor in the population and the increased risk of depression or ideation that it confers. Consequently, the highest levels of attributable risk would only be seen for risk factors that have a combination of high prevalence in the community and that confer substantially 
increased risk of depression or suicidal ideation. By taking prevalence into account, population attributable risk estimates can be used to estimate the proportion of cases of a disorder that can be attributed to a specific risk factor and to predict the potential impact of public health interventions on the health status of a population [17]. These are both important public health issues that are not adequately assessed by odds ratios alone.

The primary aim of the present study was to identify the anxiety and depression symptoms that conferred the greatest amount of risk for subsequent onset of major depressive disorder (MDD) and suicidal ideation in a community-based cohort after four year follow-up. In the present study, criteria for MDD and suicidal ideation were determined epidemiologically using the Patient Health Questionnaire (PHQ-9) [18] and the Psychiatric Symptom Frequency scale [19]. Furthermore, the study aimed to evaluate whether depression symptoms conferred more risk of developing MDD and suicidal ideation than anxiety symptoms. As the cohort consists of younger, midlife and older adults, a secondary aim was to examine whether there was age-based variation in the levels of risk attributable to these symptoms. Given the considerable focus of previous research on the role of depression symptoms in the development of depression and suicide, it was hypothesized that a greater proportion of the risk for incident depression and suicidal ideation would be attributable to depression symptoms than to anxiety symptoms. However, given the potential of anxiety to influence the onset of depression in younger adults [7], the predominance of depression effects would be lessened in the young adult cohort. It was also hypothesized that overall, depression and anxiety symptoms would more strongly account for the risk of depressive disorder and suicidal ideation in younger age groups, which tend to have more incident cases of depression and suicidal thoughts. 


\section{Method}

Participants

The PATH Through Life Project is a population-based study examining the health and well-being of people who are 20-24, 40-44, and 60-64 years of age [20]. Each cohort is being followed up every four years over a total period of 20 years. Participants were randomly sampled from the electoral rolls for the city of Canberra, Australia, and in the neighbouring town of Queanbeyan. Registration on the electoral roll is compulsory for Australian citizens. Information about the representativeness of the sample has been detailed elsewhere [21]. Results presented here concern the first two waves of interviews conducted in 1999-2002 and 2003-2006 (recruitment was staggered by age group). At the first wave, interviews were completed with 7,485 participants: 2,404 in the 20-24 year age- group, 2,530 in the 40-44 group and 2,551 in the 60-64 group. Participation rates of those who were found to be in the appropriate age ranges were $58.6 \%$ for the $20-24 \mathrm{~s}, 64.6 \%$ for the $40-44 \mathrm{~s}$ and $58.3 \%$ for the 60-64s. Four years later, interviews were completed by 6,715 participants ( $89.7 \%$ follow-up rate): 2,139 (89.0\%) aged 24-28, 2,354 (93.0\%) 44-48 and 2,222 (87.8\%) 64-68.

To ensure that analyses reflected incident cases of depression, participants who initially met criteria for depression or reported a lifetime history of depression were excluded from the analyses of the Wave 2 depression outcome. The sample selection process is shown in Figure 1. Initial criteria for depression were assessed using the Composite International Diagnostic Interview-Short Form (CIDI-SF) [22] for the 20s and 40s cohorts and the Patient Health Questionnaire (PHQ-9) [18] for the 60s cohort. Lifetime history of depression was reported using two items: "Have you ever in your life been markedly depressed; that is, for several weeks or more, you felt sad, lost interest in things and felt lacking in energy?" and "Did you see a counsellor or a doctor for it at the time?". To account for possible lifetime depression, participants were excluded if they endorsed both of these items. Further exclusion 
on the basis of missing outcome data was made, resulting in an analysis sample of 4,931 for the depression outcome that included 1,681 in the 20s cohort, 1,680 in the 40s and 1,570 in the $60 \mathrm{~s}$. For the suicidal ideation outcome, exclusions were made on the basis of suicidal ideation in the year before the first interview. Lifetime history of suicidal ideation was not assessed, due to the biases associated with long-term recall of suicidal ideation [23]. Exclusion on the basis of missing outcome data was also made, resulting in an analysis sample for the suicidal ideation outcome that included 1,561 in the 20s cohort, 1,883 in the 40s and 1,978 in the 60 s.

\section{Procedure}

Participants were interviewed at a convenient location, usually their home or the Centre for Mental Health Research at the Australian National University. Most of the interview was self-completed on a palmtop or laptop computer. However, testing by the interviewer was required for the physical tests, some cognitive tests and a cheek swab used for genetic testing. Approval for the research was obtained from The Australian National University's Human Research Ethics Committee. All participants provided written informed consent to participate in the study.

\section{Measures}

The two outcome variables were onset of major depression and onset of suicidal ideation after four years. The depression outcome was assessed using the nine-item Patient Health Questionnaire [18], a self-report measure that uses DSM criteria to assess presence of mental disorders. The criterion and construct validity of the PHQ-9 have previously been demonstrated, with $73 \%$ sensitivity and $98 \%$ specificity in detecting major depression compared to clinician-based assessment of DSM criteria and strong relationships with 
measures of functional impairment and health care use [18]. The measure also has sound internal consistency (Cronbach $\alpha>0.85$ in multiple samples) and 48-hour test-retest reliability of 0.84 [24]. Presence of major depression disorder was defined based on the algorithm identified by the authors of the scales, specifically, presence of anhedonia or feelings of depression (first two items of PHQ-9) and five or more of the nine PHQ-9 items being rated as "more than half the days" or higher (or "several days" or higher for the suicidal ideation item).

The suicidal ideation outcome was assessed broadly, based on endorsement on one or more of three yes/no items from the Psychiatric Symptom Frequency scale [19]: "In the last year have you ever felt that life is hardly worth living", "In the last year have you ever thought that you really would be better off dead?", and "In the last year have you ever thought about taking your own life?". The same criteria for the suicidal ideation outcome at wave 2 were applied to exclude participants reporting suicidal ideation at wave 1 . Despite overlaps between depression and suicidal ideation, the two outcomes had a relatively high level of independence, with a Spearman correlation of 0.27 .

The independent risk factors examined were the 18 depression and anxiety symptoms assessed in the Goldberg Depression and Anxiety Scales [25]. These scales provide binary (yes/no) self-report of symptom presence in the four weeks prior to the interview. Symptom scores from the Goldberg scales have a strong association with presence of major depression and generalised anxiety disorder, with sensitivity at specified cut points of $85 \%$ and $82 \%$ respectively and combined specificity of $91 \%$ for absence of psychiatric disorder [25]. In addition, age and gender were reported at the initial interview.

\section{Analysis}


Logistic regression analyses, adjusted for gender and initial age, were used to assess the association between each "risk factor" symptom at wave one and the onset of depression or suicidal ideation at wave two. The prevalence of each risk factor in the sample was calculated using contingency tables. Population attributable risk (PAR) was then calculated using the formula given by Fleiss [16] that takes into account both the adjusted odds of the outcome based on a single risk factor and the prevalence of that risk factor. The PAR for combined anxiety symptoms and combined depression symptoms was calculated using the method described by Bruzzi et al. [15]. Bootstrapping was conducted to estimate the standard error of the population attributable risk estimates [26]. This technique involved randomly drawing 7495 observations from the full sample of 7495 with replacement, repeating this process to create 1000 bootstrapped samples, calculating the anxiety and depression PAR for each sample and calculating a paired t-test using the 1000 bootstrapped samples. Subanalyses were conducted within each age group to assess differences in PAR across the life span, with odds ratios adjusted for gender and the symptom of interest.

\section{Results}

Sample characteristics

Characteristics of the full wave 2 sample are presented in Table 1. The p values presented in the table are based on $\chi^{2}$ statistics for categorical variables and F statistics from one-way analyses of variance for continuous variables. They show significant differences between the three age cohorts on all demographic and mental health variables. Approximately half of the sample was female and the level of education was relatively high in all age groups. Rates of depression were highest in the 20 s and lowest in the $60 \mathrm{~s}$. The increase in depression prevalence in the 20s and 40s from wave 1 to wave 2 would be 
primarily attributable to the change in instrument used in the two waves. The depression prevalence for the complete sample was $2.1 \%$ at wave 1 and $4.1 \%$ at wave 2 . Overall, some form of suicidal thought was reported by $25.8 \%$ of the 20 s cohort, $19.1 \%$ of the 40 s and $9.7 \%$ of the $60 \mathrm{~s}$ at the first wave $(18.1 \%$ overall). These rates declined in all age groups at the second wave, with $18.8 \%, 15.8 \%$ and $7.4 \%$ respectively $(14.0 \%$ overall) reporting suicidal thoughts. Of those who had depression at wave 1, 49.6\% met criteria for comorbid generalised anxiety disorder (only assessed among the 20s and 40s cohort, based on CIDI-SF, [22]) while this figure was $55.4 \%$ at wave 2 (based on the GAD-7) [27]. Comorbidity with panic disorder was assessed only in the 60 s cohort at wave 1 and in the full cohort at wave 2 based on the PHQ-panic [18], with rates of $15.2 \%$ and $21.0 \%$ respectively among those with depression.

\section{Incidence of depression attributable to anxiety and depression symptoms}

Table 2 shows the estimates of population attributable risk (PAR) for onset of depression after four years, based on the symptoms assessed at the first wave. The table also shows the odds ratios adjusted for age cohort and gender, and the prevalence of each symptom in the cohort. All adjusted odds ratios were significant at $p<0.05$, indicating that each of the symptoms was associated with greater odds of depression. The presentation of anxiety and depression items in the table are sorted based on PAR. It is clear from the table that anxiety symptoms, in particular, worrying, irritability and difficulty relaxing, have the largest PARs as a result of their strong relationships with depression and relatively high prevalence in the sample. Overall, $45.1 \%$ of the incident cases of major depression at wave 2 were attributable to anxiety symptoms, while $34.8 \%$ were attributable to depression symptoms. Bootstrapping was used to estimate the mean difference between anxiety and depression symptom PARs (mean difference $=0.109$ ), and the standard error of this estimate 
$(\mathrm{SE}=0.026)$, by calculating these PARs across 1000 bootstrapped samples which were sampled from the full cohort $(n=7495)$ with replacement. Based on a paired t-test, the difference between the estimated PARs for anxiety symptoms and depression symptoms was significant $\left(\mathrm{t}_{4930}=4.22, \mathrm{p}<0.001\right)$.

In a follow-up analysis (not tabulated), the PARs were recalculated excluding participants with GAD at baseline, but only among the 20s and 40s samples for whom GAD criteria were assessed using the CIDI-SF. Although there was some attenuation of both PARs after the additional exclusion, anxiety symptoms still accounted for more risk of depression onset than did depression symptoms: $40.2 \%$ for anxiety symptoms and $29.5 \%$ for depression symptoms (compared to $58.0 \%$ and $43.0 \%$ respectively for the combined 20 s and 40 s cohorts when only depression was excluded).

These findings are broken down by age cohort in Figure 2. Again, worrying, irritability and difficulty relaxing stand out as having the largest PARs, with worrying accounting for $72 \%$ of the risk for depression incidence in the 20 s cohort. However, there were some distinct age differences. Presence of headaches or neck aches featured prominently for the 40 s and 60 s cohorts ( $48.5 \%$ and $57.3 \%$ respectively). Beyond headaches and neck aches, the symptoms that contributed most to depression incidence in the 60 s cohort were lack of energy (44.9\%), worries about health (39.5\%) and difficulty concentrating (37.5\%). As summarised in Table 3, anxiety and depression symptoms accounted for the greatest proportion of the incident depression cases in the 20s, and the least in the $60 \mathrm{~s}$. Likewise, the difference in PAR between anxiety symptoms and depression symptoms was pronounced in the $20 \mathrm{~s}$ and $40 \mathrm{~s}$ but marginal in the $60 \mathrm{~s}$. Note that the adjusted PAR for waking early in the 60 s was negative $(-13.1 \%)$.

Incidence of suicidal ideation attributable to anxiety and depression symptoms 
The last column of Table 2 shows the estimates of PAR for onset of suicidal ideation after four years, based on the symptoms assessed at the first wave. All adjusted odds ratios were significant at $p<0.05$ except for waking early $(p=0.36)$. The table shows that once again, the incidence of suicidal ideation attributable to anxiety symptoms ( $24.3 \%$ overall) was greater than that attributable to depression symptoms (16.1\% overall). Irritability, feeling keyed up and worrying had the greatest PARs, which reflect odds ratios greater than 2 and symptom prevalence above $30 \%$ for these three symptoms. The magnitude of the PARs for suicidal ideation was less than those for depression, indicating that a smaller proportion of the incidence of suicidal ideation was attributable to depression and anxiety symptoms than for depression incidence. Using the bootstrap method, the mean difference between the PARs for anxiety and depression symptoms $(0.062, \mathrm{SE}=0.023)$ on suicidal ideation was significant $\left(t_{5231}=2.70, p=0.007\right)$. To examine whether the risk estimates were related to the low threshold used to characterise suicidal thoughts, the analyses were repeated with ideation assessed using only the item "In the last year have you ever thought about taking your own life?". Participants reporting any level of suicidal thoughts at the first wave were once again excluded. In this follow-up analysis (not tabulated), the PARs for anxiety symptoms and depression symptoms were similar to the original analysis: $22.3 \%$ and $11.0 \%$ respectively.

Figure 3 plots the age-specific PARs for suicidal ideation. In contrast to depression, the 40s cohort had larger PARs than the 20s or 60s. In the 20s cohort, difficulty falling asleep had a stronger impact on suicidal ideation (23.2\%) than in other age cohorts, with irritability (31.0\%) being the only symptom with a greater PAR. The 40s cohort aligned with the overall findings, with feeling keyed up or irritable contributing more than $40 \%$ of the incidence of suicidal ideation. In the 60s cohort, feeling slowed up (37.6\%), headaches or neck aches (29.9\%) and lacking in energy $(23.9 \%)$ were the symptoms most strongly contributing to suicidal ideation. Note that the PARs for waking early in the $20 \mathrm{~s}(-2.1 \%)$ and lost weight in 
the 60 s $(-1.4 \%)$ were negative. The age differences observed in Figure 3 are also evident in Table 3, which shows that the 40s cohort had higher PARs overall for both depression and anxiety symptoms than the other two age cohorts on the suicidal ideation outcome. Table 3 also indicates that the difference between the PARS for anxiety and depression symptoms was most narrow in the 60 s cohort.

\section{Discussion}

The present study examined the proportion of the incidence of depression and suicidal ideation that was attributable to anxiety and depression symptoms in a cohort of approximately 5,000 community-dwelling adults. Contrary to hypotheses, anxiety symptoms were found to account for a larger proportion of depression incidence and suicidal ideation incidence than depression symptoms. Although anxiety symptoms tended to be slightly more prevalent in the cohort, many of them conferred greater odds of depression and/or suicidal ideation than the depression symptoms. Consequently, it appears that programs to prevent depression and suicide in the community may benefit from aiming to reduce anxiety symptoms in addition to depression symptoms. In particular, targeted depression programs may be more successful if they select individuals reporting symptoms such as worrying, irritability or difficulty relaxing, which may be overlooked by programs that define risk based on severity of depression symptoms. Likewise, targeted suicide prevention programs may benefit from selecting individuals who have signs of anxiety, particularly symptoms of agitation such as irritability, feeling keyed-up or worrying. These symptoms may be missed if depression severity is used as the criterion for entry into a targeted suicide prevention program. Indeed, core depression symptoms including loss of interest and feelings of hopelessness had only moderate impact on depression incidence and minor impact on the development of suicidal thoughts, although this was partly due to the low prevalence of these 
symptoms in the community. While hopelessness is a strong risk factor for suicidal ideation [28], universal suicide prevention interventions that aim to reduce worrying and irritability may be more effective than interventions to reduce hopelessness.

The symptoms that had the greatest impact on depression incidence also tended to have a larger impact on suicidal ideation incidence, although the overall impact of mental health symptoms was less for suicidal ideation. It is likely that there is a greater range of determinants of suicidal ideation than depression, many of which may be beyond the realm of mental health symptoms. Emergence of suicidal ideation may also be less predictable than onset of depression. Nevertheless, the patterns of attributable risk for both depression and suicidal ideation were quite consistent within age cohorts. Physical symptoms such as headaches and neck aches and lacking energy accounted for a greater proportion of depression and suicidal ideation incidence in the older cohort. This finding may be reflective of depression being expressed differently across the lifespan, with physical health becoming an important determinant of mental wellbeing in later life [see 29]. Age-related changes in the determinants of depression may also explain why the differences between the attributable risk for anxiety and depression symptoms narrowed in the older cohort. Poor sleep in general had a moderate to large impact on depression incidence, although the effects were confined to younger cohorts and more specific sleep problems such as early wakefulness and difficulty falling asleep had much smaller impacts.

The mechanisms for the effects of anxiety symptoms on depression and suicidal ideation warrant further research. The findings may partly be explained by developmental or time course explanations. Specifically, "pure" depression may manifest earlier than comorbid anxiety and depression, as anxiety tends to occur earlier than depression [7]. However, a similar proportion of individuals who met criteria for depression at wave 1 had comorbid generalised anxiety compared to those who had incident depression at wave 2 (50\% at wave 1 
vs. $55 \%$ at wave 2), so this explanation does not fully account for the stronger effects of anxiety. Furthermore, the difference between PARs remained after accounting for initial presence of GAD in the 20 s and 40s cohorts. Another explanation could be that the increased PARs for anxiety symptoms simply reflect that anxiety symptoms as measured by the Goldberg scale are more common than the depression symptoms. However, the odds ratios for anxiety symptoms were comparable to those for the depression symptoms. The effects of anxiety symptoms on suicidality are more consistent with the literature, which shows strong evidence that individuals with anxiety disorders are at increased risk of suicidal ideation [10]. Theoretical models have tended to distinguish depression as the primary determinant of suicidal thoughts but anxiety and agitation as stronger determinants for the propensity to enact suicidal behaviours [10]. However there is growing evidence for the importance of anxiety in the development of suicidal ideation $[9 ; 10 ; 30 ; 31]$. Investigation into common neurobiological pathways involved in anxiety, depression and suicidality may shed further light on the observed relationships [32].

While there were many strengths of the current study, including the large populationbased dataset, the use of incidence outcome measures and the examination of a wide range of symptoms across adulthood, some limitations to the findings should be noted. Prior episodes of depression and suicidal ideation may have been missed, particularly from the period long before the wave 1 interview. Participants who had experienced an episode of depression may not have sought help from a doctor. Conversely, some of the reported lifetime depression episodes may not have met clinical criteria. Likewise, the suicidal ideation measure did not take into account the frequency or severity of the ideation and may have been subject to recall biases. Nevertheless, the suicidal ideation findings remained when only overt thoughts of suicide were examined. A full clinical interview may have been more accurate in assessing the depression and suicidality outcomes, although such an assessment was not feasible in the 
context of a large epidemiological study. Assessments were only completed every four years, with reports on suicidality based on the year prior to assessment. The episodic nature of suicidality and depression may have resulted in missed episodes and underestimates of risk. Finally, additional analyses that account for a range of other risk factors, such as physical health, social support, substance use, personality and genetic markers may lead to a better understanding of the mechanisms underlying the observed relationships.

\section{Conclusion}

Based on the findings in the present cohort, more of the incidence of depression and suicidal ideation was attributable to anxiety symptoms than depression symptoms at the population level. Community-based prevention programs may increase their effectiveness if they target individuals experiencing symptoms of anxiety including worry, irritability and difficulty relaxing. Such programs should also consider aiming to reduce anxiety symptoms in addition to depression symptoms. Different patterns of risk across adulthood also indicate that prevention programs that are tailored to individual circumstances, such as age, may be more successful than generic programs. 


\section{References}

1. Georgiades K, Lewinsohn PM, Monroe SM, Seeley JR. Major depressive disorder in adolescence: the role of subthreshold symptoms. J Am Acad Child Adolesc Psychiatry 2006;45(8):936-44.

2. Kessler RC, Zhao S, Blazer DG, Swartz M. Prevalence, correlates, and course of minor depression and major depression in the National Comorbidity Survey. J Affect Disord 1997;45(1-2):19-30.

3. Joiner TE, Jr., Brown JS, Wingate LR. The psychology and neurobiology of suicidal behavior. Annu Rev Psychol 2005;56:287-314.

4. Kovacs M, Lopez-Duran N. Prodromal symptoms and atypical affectivity as predictors of major depression in juveniles: implications for prevention. J Child Psychol Psychiatry 2010;51(4):472-96.

5. Mathew AR, Pettit JW, Lewinsohn PM et al. Co-morbidity between major depressive disorder and anxiety disorders: shared etiology or direct causation? Psychol Med 2011;41(10):2023-34.

6. Kessler RC, Nelson CB, McGonagle KA et al. Comorbidity of DSM-III-R major depressive disorder in the general population: results from the US National Comorbidity Survey. Br J Psychiatry Suppl 1996;(30):17-30.

7. Wittchen HU, Kessler RC, Pfister H, Lieb M. Why do people with anxiety disorders become depressed? A prospective-longitudinal community study. Acta Psychiatr Scand Suppl 2000;(406):14-23.

8. Coryell W, Endicott J, Winokur G. Anxiety syndromes as epiphenomena of primary major depression: outcome and familial psychopathology. Am J Psychiatry 1992;149(1):100-7. 
9. Cougle JR, Keough ME, Riccardi CJ, Sachs-Ericsson N. Anxiety disorders and suicidality in the National Comorbidity Survey-Replication. J Psychiatr Res 2009;43(9):825-9.

10. Nock MK, Hwang I, Sampson N et al. Cross-national analysis of the associations among mental disorders and suicidal behavior: findings from the WHO World Mental Health Surveys. PLoS Med 2009;6(8):e1000123.

11. Khan A, Leventhal RM, Khan S, Brown WA. Suicide risk in patients with anxiety disorders: a meta-analysis of the FDA database. J Affect Disord 2002;68(2-3):183-90.

12. Bronisch T, Wittchen HU. Suicidal ideation and suicide attempts: comorbidity with depression, anxiety disorders, and substance abuse disorder. Eur Arch Psychiatry Clin Neurosci 1994;244(2):93-8.

13. Fawcett J, Scheftner WA, Fogg L et al. Time-related predictors of suicide in major affective disorder. Am J Psychiatry 1990;147(9):1189-94.

14. Fava M, Alpert JE, Carmin CN et al. Clinical correlates and symptom patterns of anxious depression among patients with major depressive disorder in STAR *D. Psychol Med 2004;34(7):1299-308.

15. Bruzzi P, Green SB, Byar DP et al. Estimating the population attributable risk for multiple risk factors using case-control data. Am J Epidemiol 1985;122(5):904-14.

16. Fleiss JL. Inference about population attributable risk from cross-sectional studies. Am J Epidemiol 1979;110(2):103-4.

17. Northridge ME. Public health methods--attributable risk as a link between causality and public health action. Am J Public Health 1995;85(9):1202-4.

18. Spitzer RL, Kroenke K, Williams JB. Validation and utility of a self-report version of PRIME-MD: the PHQ primary care study. Primary Care Evaluation of Mental Disorders. Patient Health Questionnaire. Jama 1999;282(18):1737-44. 
19. Lindelow M, Hardy R, Rodgers B. Development of a scale to measure symptoms of anxiety and depression in the general UK population: the psychiatric symptom frequency scale. J Epidemiol Community Health 1997;51(5):549-57.

20. Anstey KJ, Christensen H, Butterworth P et al. Cohort Profile: The PATH through life project. International journal of epidemiology 2012;41(4):951-960.

21. Rodgers B, Windsor TD, Anstey KJ et al. Non-linear relationships between cognitive function and alcohol consumption in young, middle-aged and older adults: the PATH Through Life Project. Addiction 2005;100(9):1280-90.

22. Kessler RC, Andrews G, Mroczek D et al. The World Health Organization Composite International Diagnostic Interview Short Form (CIDI-SF). International Journal of Methods in Psychiatric Research 1998;7:171-185.

23. Klimes-Dougan B, Safer MA, Ronsaville D et al. The value of forgetting suicidal thoughts and behavior. Suicide Life Threat Behav 2007;37(4):431-8.

24. Kroenke K, Spitzer RL, Williams JB. The PHQ-9: validity of a brief depression severity measure. J Gen Intern Med 2001;16(9):606-13.

25. Goldberg D, Bridges K, Duncan-Jones P, Grayson D. Detecting anxiety and depression in general medical settings. Bmj 1988;297(6653):897-9.

26. Efron B, Tibshirani RJ. An Introduction to the Bootstrap. Boca Raton, FL: Chapman and Hall/CRC. 1994.

27. Spitzer RL, Kroenke K, Williams JB, Lowe B. A brief measure for assessing generalized anxiety disorder: the GAD-7. Arch Intern Med 2006;166(10):1092-7.

28. Joiner TE, Jr., Rudd MD. Disentangling the interrelations between hopelessness, loneliness, and suicidal ideation. Suicide Life Threat Behav 1996;26(1):19-26. 
29. Roberts RE, Kaplan GA, Shema SJ, Strawbridge WJ. Prevalence and correlates of depression in an aging cohort: the Alameda County Study. J Gerontol B Psychol Sci Soc Sci 1997;52(5):S252-8.

30. Hawgood J, De Leo D. Anxiety disorders and suicidal behaviour: an update. Current opinion in psychiatry 2008;21(1):51-64.

31. Batterham PJ, Christensen H. Longitudinal risk profiling for suicidal thoughts and behaviours in a community cohort using decision trees. J Affect Disord In press.

32. Mann JJ. The neurobiology of suicide. Nat Med 1998;4(1):25-30. 
Table 1: Descriptive statistics for the complete Wave 2 sample $(\mathrm{N}=6715)$

\begin{tabular}{|c|c|c|c|c|c|c|c|c|c|}
\hline & \multirow[b]{2}{*}{$\mathrm{N}$} & \multicolumn{2}{|c|}{$\begin{array}{l}\text { 20s cohort } \\
(\mathrm{N}=2,139)\end{array}$} & \multicolumn{2}{|c|}{$\begin{array}{l}\text { 40s cohort } \\
(\mathrm{N}=2,354)\end{array}$} & \multicolumn{2}{|c|}{$\begin{array}{l}60 \text { s cohort } \\
(\mathrm{N}=2,222)\end{array}$} & \multirow[b]{2}{*}{$x^{2}$} & \multirow[b]{2}{*}{$\mathrm{p}$} \\
\hline & & Frequency & Percent & Frequency & Percent & Frequency & Percent & & \\
\hline Female gender & 6715 & 1126 & $52.6 \%$ & 1251 & $53.1 \%$ & 1075 & $48.4 \%$ & 12.30 & 0.002 \\
\hline Married / de facto relationship, wave 1 & 6698 & 509 & $24.0 \%$ & 1869 & $79.4 \%$ & 1758 & $79.2 \%$ & 1882.57 & $<0.001$ \\
\hline Current depression, wave 1 & 6684 & 56 & $2.6 \%$ & 54 & $2.3 \%$ & 33 & $1.5 \%$ & 7.28 & 0.026 \\
\hline Lifetime depression, wave 1 & 6681 & 405 & $19.1 \%$ & 636 & $27.2 \%$ & 605 & $27.3 \%$ & 51.83 & $<0.001$ \\
\hline Current depression, wave 2 & 6639 & 127 & $6.0 \%$ & 104 & $4.5 \%$ & 42 & $1.9 \%$ & 46.08 & $<0.001$ \\
\hline \multicolumn{10}{|l|}{ Suicidal ideation items } \\
\hline Life not worth living, wave 1 & 6680 & 493 & $23.2 \%$ & 410 & $17.5 \%$ & 196 & $8.8 \%$ & 166.34 & $<0.001$ \\
\hline Better off dead, wave 1 & 6680 & 311 & $14.7 \%$ & 262 & $11.2 \%$ & 110 & $5.0 \%$ & 114.68 & $<0.001$ \\
\hline Thought about taking life, wave 1 & 6681 & 268 & $12.6 \%$ & 200 & $8.5 \%$ & 63 & $2.8 \%$ & 143.58 & $<0.001$ \\
\hline Life not worth living, wave 2 & 6659 & 373 & $17.5 \%$ & 351 & $15.0 \%$ & 154 & $7.0 \%$ & 114.49 & $<0.001$ \\
\hline Better off dead, wave 2 & 6662 & 254 & $11.9 \%$ & 207 & $8.8 \%$ & 90 & $4.1 \%$ & 88.80 & $<0.001$ \\
\hline \multirow[t]{2}{*}{ Thought about taking life, wave 2} & 6656 & 204 & $9.6 \%$ & 154 & $6.6 \%$ & 48 & $2.2 \%$ & 105.14 & $<0.001$ \\
\hline & $\mathrm{N}$ & Mean & SD & Mean & SD & Mean & SD & $\mathrm{F}$ & $\mathrm{p}$ \\
\hline Years of education & 6574 & 14.24 & 1.49 & 14.44 & 2.27 & 13.93 & 2.63 & 30.37 & $<0.001$ \\
\hline PHQ-9 depression score & 6639 & 5.10 & 4.63 & 3.99 & 4.30 & 2.48 & 3.22 & 220.95 & $<0.001$ \\
\hline
\end{tabular}

Notes: Current depression assessed using CIDI-SF in 20s \& 40s cohorts at wave 1, PHQ-9 in 60s cohort at wave 1 and PHQ-9 in all cohorts at wave 2; $p$ values are based on $\chi^{2}$ statistics for categorical variables and $F$ statistics from one-way analyses of variance for continuous variables 
Table 2: Population attributable risk for the onset of depression $(n=4,931)$ and suicidal ideation $(n=5,422)$ after four years based on anxiety and depression symptoms

\begin{tabular}{|c|c|c|c|c|c|c|}
\hline & \multicolumn{3}{|c|}{ Depression onset $(n=4,931)$} & \multicolumn{3}{|c|}{ Suicidal ideation onset $(n=5,422)$} \\
\hline & $\begin{array}{l}\text { Adjusted } \\
\text { odds ratio }\end{array}$ & Prevalence & $\begin{array}{r}\text { Population } \\
\text { Attributable Risk }\end{array}$ & $\begin{array}{l}\text { Adjusted } \\
\text { odds ratio }\end{array}$ & Prevalence & $\begin{array}{r}\text { Population } \\
\text { Attributable Risk }\end{array}$ \\
\hline Anxiety symptoms & & & $45.1 \%$ & & & $24.3 \%$ \\
\hline worrying & 4.91 & $33.7 \%$ & $56.9 \%$ & 2.07 & $31.4 \%$ & $25.1 \%$ \\
\hline irritable & 4.08 & $36.3 \%$ & $52.8 \%$ & 2.34 & $34.2 \%$ & $31.4 \%$ \\
\hline difficulty relaxing & 4.47 & $30.4 \%$ & $51.3 \%$ & 1.82 & $28.7 \%$ & $19.0 \%$ \\
\hline sleeping poorly & 3.70 & $32.2 \%$ & $46.5 \%$ & 1.63 & $31.3 \%$ & $16.5 \%$ \\
\hline headaches/neck aches & 2.84 & $41.6 \%$ & $43.3 \%$ & 1.71 & $42.1 \%$ & $23.1 \%$ \\
\hline keyed-up & 2.94 & $33.8 \%$ & $39.6 \%$ & 2.17 & $33.9 \%$ & $28.4 \%$ \\
\hline difficulty falling asleep & 3.46 & $23.9 \%$ & $37.0 \%$ & 1.63 & $23.0 \%$ & $12.7 \%$ \\
\hline worried about health & 3.19 & $25.4 \%$ & $35.7 \%$ & 1.82 & $24.1 \%$ & $16.5 \%$ \\
\hline somatic symptoms & 3.32 & $20.9 \%$ & $32.7 \%$ & 1.63 & $20.1 \%$ & $11.3 \%$ \\
\hline Depression symptoms & & & $34.8 \%$ & & & $16.1 \%$ \\
\hline felt slowed up & 3.95 & $28.4 \%$ & $45.6 \%$ & 1.85 & $27.7 \%$ & $19.0 \%$ \\
\hline difficulty concentrating & 3.99 & $26.6 \%$ & $44.3 \%$ & 2.18 & $24.2 \%$ & $22.2 \%$ \\
\hline lacking energy & 3.20 & $33.1 \%$ & $42.1 \%$ & 1.70 & $32.5 \%$ & $18.6 \%$ \\
\hline lost interest & 5.29 & $16.4 \%$ & $41.4 \%$ & 1.69 & $12.9 \%$ & $8.2 \%$ \\
\hline felt hopeless & 6.03 & $11.0 \%$ & $35.6 \%$ & 2.55 & $7.2 \%$ & $10.0 \%$ \\
\hline lost confidence & 5.26 & $12.7 \%$ & $35.1 \%$ & 1.91 & $9.9 \%$ & $8.2 \%$ \\
\hline feel worse in mornings & 2.95 & $21.8 \%$ & $29.9 \%$ & 1.61 & $20.0 \%$ & $10.8 \%$ \\
\hline waking early & 1.46 & $42.4 \%$ & $16.2 \%$ & 1.10 & $42.3 \%$ & $4.1 \%$ \\
\hline lost weight & 3.66 & $4.7 \%$ & $11.1 \%$ & 1.56 & $3.7 \%$ & $2.0 \%$ \\
\hline
\end{tabular}

Note: Symptoms are ordered based on PAR for depression; "somatic symptoms" indicates presence of trembling, tingling, dizzy spells, sweating, diarrhoea, or needing to pass water more often than usual 
Table 3: Population attributable risk for the onset of depression and suicidal ideation by age cohort, based on overall anxiety symptoms and overall depression symptoms

\begin{tabular}{lcccccc}
\hline & \multicolumn{3}{c}{ Depression at wave 2 } & \multicolumn{3}{c}{ Suicidal ideation at wave 2 } \\
& $20 \mathrm{~s}$ & $40 \mathrm{~s}$ & $60 \mathrm{~s}$ & $20 \mathrm{~s}$ & $40 \mathrm{~s}$ & $60 \mathrm{~s}$ \\
\hline Anxiety symptoms at wave 1 & $51.5 \%$ & $40.1 \%$ & $25.7 \%$ & $16.9 \%$ & $28.3 \%$ & $15.1 \%$ \\
Depression symptoms at wave 1 & $38.5 \%$ & $28.9 \%$ & $21.5 \%$ & $5.9 \%$ & $17.2 \%$ & $12.7 \%$ \\
\hline
\end{tabular}


Figure 1: Selection of participants into the analyses

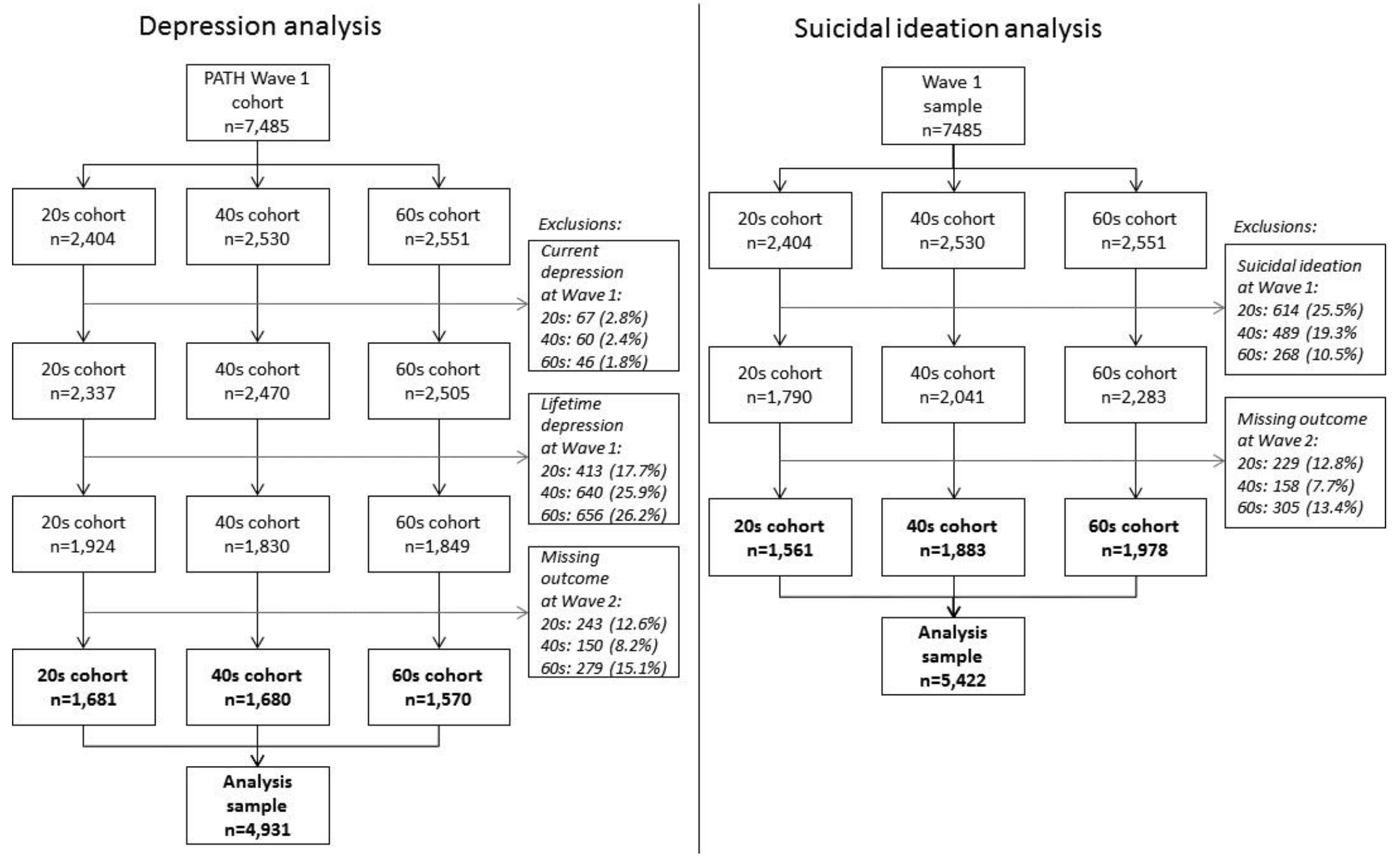


Figure 2: Gender-adjusted population attributable risk for onset of depression by age cohort

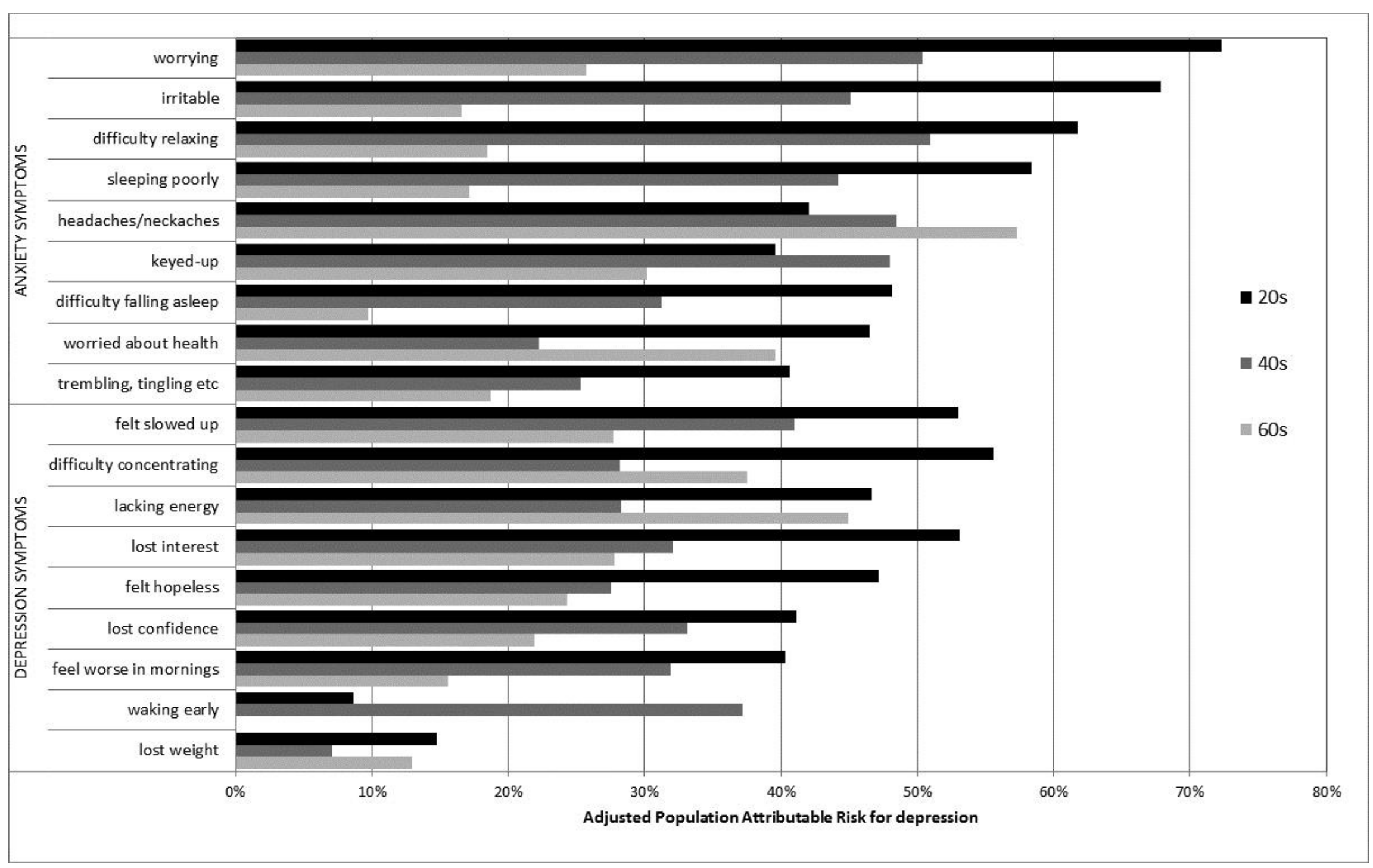


Figure 3: Gender-adjusted population attributable risk for onset of suicidal ideation by age cohort

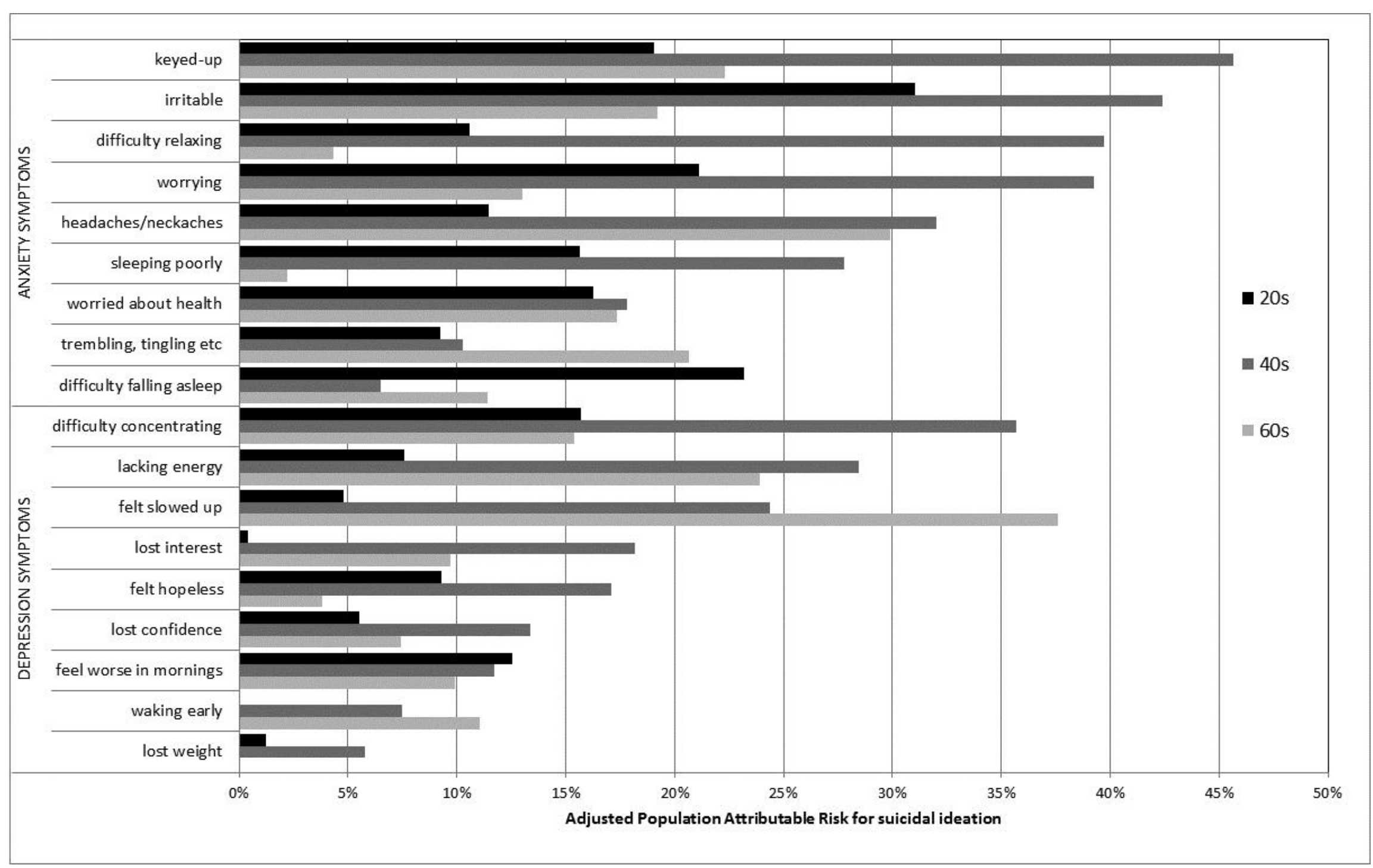

